\title{
Editorial
}

\section{A Half-Century of CMP}

\author{
Horng-Tzer Yau \\ Cambridge, USA. E-mail: htyau@math.harvard.edu
}

Published online: 30 August 2016 - C Springer-Verlag Berlin Heidelberg 2016

Fifty-one years ago, Communications in Mathematical Physics published its first issue. This special moment marked the beginning of what has become the top journal in mathematical physics, a home for the great works produced by our community. CMP sprang up out of the energetic efforts of a small but devoted group of mathematical physicists who believed in the need for a journal in which physics and mathematics were both of central interest. This group included Res Jost and Rudolf Haag, who both played key roles in creating the journal. Haag served as the journal's first editor-in-chief, and its initial editorial board consisted of N. Hugenholtz, D. Ruelle, L. Schwartz, and A. Taub. Haag served as chief editor for eight years, and during his tenure the journal published the highest quality works in mathematical physics, setting the standard for all the editors who have followed.

A critical factor in the journal's consistency and excellence over the years has been its editors' commitment to the original vision for the journal. This commitment is reflected in the longstanding tenures of many of CMP's chief and associate editors. After Haag (1965-1973), K. Hepp (1973-1976), J. Glimm (1976-1978), A. Jaffe (1978-2000), and M. Aizenman (2000-2012) followed as chief editors. H. Araki, J. Lebowitz, and B. Simon served remarkably as editors for more than three decades; A. Connes, M. Fisher, and Y. Sinai served for more than two decades. Other editors who have served for many years include G. Gallavotti, A. Kupiainen, and S. T. Yau. Members of CMP's advisory board who have served many years include M. F. Atiyah, J. Cardy, L. Faddeev, J. Fröhlich, F. Hirzebruch, G. 't Hooft, N. Seiberg, R. Schrieffer, I. M. Singer, and C. N. Yang.

As its name suggests, CMP is a journal communicated by its board members. The members of the editorial board work closely together to ensure the standard and quality of the journal, handling all editorial work and making decisions on submissions within their respective areas of interest. This work serves as the backbone of the journal and requires a great deal of commitment. CMP is deeply appreciative of the dedicated efforts of all of our editors and advisory board members, including the many we are unable to list 
here. It is thanks to their work that CMP has become the journal we know and celebrate today.

CMP is also indebted to the numerous reviewers who have worked on behalf of the journal and the mathematical physics community. This selfless and anonymous work, which is often a laborious and time-intensive task, is done in service to the journal and the community. It is an indispensable part of the scientific process and provides a tremendous benefit to the authors. Many of CMP's published works have been greatly improved upon thanks to the contributions of our referees.

Of course, CMP would be unrecognizable today if it were not for the tremendous support it has enjoyed from Springer over the years. Springer's editors have worked hand in hand with CMP's editorial board from the very start. Thanks to this strong partnership CMP has flourished and has provided its readership with a journal of the highest standards. The Springer editors who have served in this capacity include Hermann Mayer-Kaupp, Wolf Beiglöck, Liesbeth Mol, and Aldo Rampioni (CMP's current managing editor). We wish to also express our gratitude to all of CMP's supporting staff, especially Barbara Drauschke, Herma Drees, Regina Finn, Alexander Gontar, Astrid Huizer, Sabine Lehr, Andrea Klueber, Mahalakshmi Mariappan, Pasumpon Mayilvaganan, and Kirsten Theunissen. Authors, editors, and referees have greatly benefited from their assistance and contributions.

While CMP has maintained a remarkable degree of continuity over the past five decades, it has undergone many changes between 1965 and today that reflect the growth and vibrancy of the mathematical physics community. Today CMP publishes on average nearly 250 papers per year, a dramatic increase when compared to the 49 published in the entirety of 1966, CMP's first full year. The field of mathematical physics itself also continues to evolve, and consequently the topics covered by CMP have changed substantially over time.

Nevertheless, the journal has never deviated from its goal of publishing physically relevant papers with ingenious mathematical ideas, and demanding mathematical proof when theorems are claimed. All CMP editors share the belief that new mathematical methods are often discovered through studying physics and that new mathematical advancements can frequently play key roles in novel physics theories. In this respect, CMP is unique among all mathematics and physics journals. As CMP moves forward into its next fifty years, we sincerely hope that this tradition will continue and inspire many new and important advances in mathematics and physics in the years to come.

Horng-Tzer Yau

On behalf of the editorial board 